\title{
Laboratory characterization of the ARGOS laser wavefront sensor
}

\author{
Marco Bonaglia ${ }^{a}$, Lorenzo Busoni ${ }^{a}$, Luca Carbonaro ${ }^{a}$, Fernando Quirós Pacheco ${ }^{a}$, Marco \\ Xompero $^{a}$, Simone Esposito $^{a},{\text { Gilles } \text { Orban }^{b} \text { de Xivry, and Sebastian Rabien }}^{b}$ \\ ${ }^{a}$ Osservatorio Astrofisico di Arcetri, L.go E. Fermi 5, 50125 Firenze, Italy; \\ ${ }^{b}$ Max-Planck-Institut für extraterrestrische Physik, Giessenbachstraße, 85748 Garching, \\ Germany
}

\begin{abstract}
In this paper we present the integration status of the ARGOS wavefront sensor and the results of the closed loop tests performed in laboratory. ARGOS is the laser guide star adaptive optics system of the Large Binocular Telescope. It is designed to implement a Ground Layer Adaptive Optics correction for LUCI, an infrared imaging camera and multi-object spectrograph, using 3 pulsed Rayleigh beacons focused at $12 \mathrm{~km}$ altitude. The WFS is configured as a Shack-Hartman sensor having $15 \times 15$ subaspertures over the telescope pupil. In the WFS each LGS is independently stabilized for on-sky jitter and range-gated to reduce spot elongation. The 3 LGS are arranged on a single lenslet array and detector by the use of off-axis optics in the final part of the WFS.

The units of WFS are in the integration and testing phase at Arcetri Observatory premises. We describe here the test aimed to demonstrate the functionality of the WFS in an adaptive optics closed loop performed using the internal light source of the WFS and a MEMS deformable mirror.
\end{abstract}

Keywords: Large Binocular Telescope, adaptive optics, laser guide star, Shack-Hartmann wavefront sensor, MEMS.

\section{INTRODUCTION}

ARGOS is the LBT laser guide star adaptive optics system. At first it will implement a Ground Layer AO (GLAO) correction over a wide field of view, reducing the natural seeing typical of the LBT site of a factor two or better, bringing to a significative gain in terms of science throughput obtainable with the LBT instrumentation. ${ }^{1}$ ARGOS has been designed mainly to serve the 2 LUCI instruments, near infrared multi-object spectrographs and imaging cameras having a field of view of $4 \times 4^{\prime}{ }^{2}$ Since LUCIs are single beam instruments, ARGOS will be replicated for both eyes of the telescope. ARGOS will produce a constellation of 3 Rayleigh beacons at $532 \mathrm{~nm}$ emitted by Nd:Yag pulsed lasers. ${ }^{4}$ The 3 laser guide stars, expanded and focussed at $12 \mathrm{~km}$ altitude by a refractive launch system, will be arranged on a triangle inscribed in a $2^{\prime}$ radius circle. A dedicated laser WaveFront Sensor $(\text { LGS-WFS })^{3}$ will allow to perform a tomographic measurement of the ground layer turbulence. In the WFS the cone of atmosphere traversed by each LGS will be independently sensed using a Shack-Hartmann (SH) sensor having 15 subapertures over the pupil diameter. Slopes are computed independently for the 3 sensors by a Basic Computational Unit $(\mathrm{BCU})^{5}$ using a centroiding algorithm. The wavefront aberrations due to the ground layer turbulence are then reconstructed on board of the LBT Adaptive Secondary BCU and the AO correction is applied at $1 \mathrm{kHz}$ rate as a set of 150 modes by the ASM thin shell. ${ }^{6}$

The LGS-WFS unit will be installed next to the LUCI focal station, as shown in figure 1. A large dichroic window installed just in front of the instrument focal station will split the light at a $600 \mathrm{~nm}$ transmitting both the red wavelengths to the atmospheric tip-tilt sensor, made of a quad-cell sensor placed onboard of the First Light Adaptive Optics (FLAO) WFS unit, ${ }^{11}$ and the infrared wavelengths to LUCI. Lasers light will be reflected by the dichroic and directed toward the LGS-WFS by an auxiliary deployable folding mirror.

Figure 1 shows the optomechanical structure of the LGS-WFS installed a side of LUCI focal station. The internal layout of the LGS-WFS will be described in detail in section 2.

Two units of the LGS-WFS are now assembled and tested at Arcetri premises. To perform the test their internal

Send correspondence to: Marco Bonaglia, E-mail: mbona@arcetri.astro.it, Telephone: +39055 2752303

Adaptive Optics Systems III, edited by Brent L. Ellerbroek, Enrico Marchetti, Jean-Pierre Véran, Proc. of SPIE Vol. 8447, 84476B · @ 2012 SPIE · CCC code: 0277-786/12/\$18 · doi: 10.1117/12.925742 

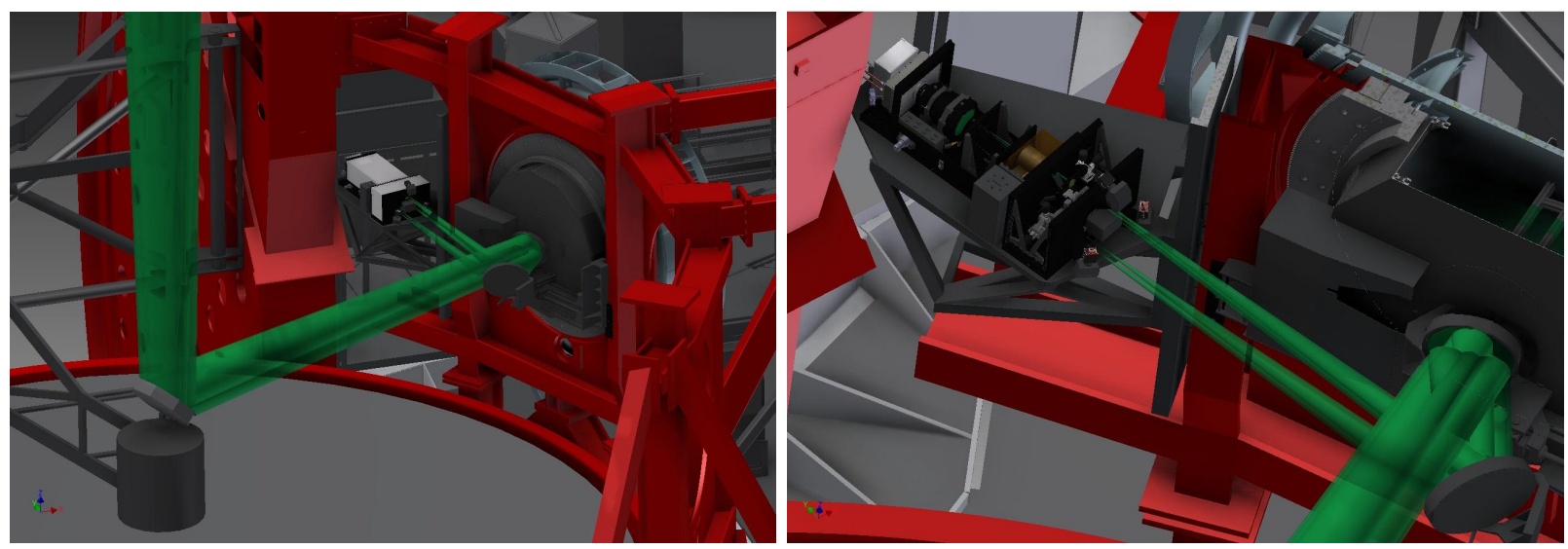

Figure 1. Left: model of the LBT instrumentation gallery showing the LGS-WFS installed next to the LUCI focal station. The green beams show the laser light coming from the secondary, reflected by the tertiary and finally directed toward the LGS-WFS by the combination of a dichroic window and a deployable fold mirror. Right: close up view of the LGS-WFS having the enclosure removed to better show the internal optomechanical arrangement.

light source have been equipped with a MEMS-DM to act both as corrector and as turbulence generator. The test setup and the calibration procedure employed to drive the DM in open loop are described in section 3. Section 4 describes the test performed in laboratory and resumes the LGS-WFS performance measured in the closed loop test.

\section{LGS-WFS LAYOUT}

The LGS-WFS is composed of three main parts: the front one contains 3 independent sets of optics (one for each LGS) devoted to stabilize and range-gate the laser beams. A second part contains some relay off-axis optics to re-image properly the pupil plane on the lenslet array and to fine-tune the position of the spot pattern on the detector. The final part of the device performs the wavefront sensing that is carried out arranging the 3 beams on a single Lenslet array (LA) and detector. In addition to that, the WFS is provided with a internal calibration unit that is fitted in the front part of the WFS in between the 3 LGS beams optics. Finally, three acquisition cameras patrol a $1^{\prime}$ field to measure the on-sky position of the LGS and to recenter them within the $4.7^{\prime \prime}$ FoV of the LGS-WFS working in close loop with the laser pointing system.

Figure 2 shows the arrangement of the WFS optics. Following the path of the light coming from the telescope on the left, the first optic of the WFS is a $100 \mathrm{~mm}$ collimator (1) placed on a remotely controlled XY stage. This stage is used to stabilize the beam position on the LA plane allowing to compensate for any misalignment introduced in the system by mechanical flexures of the telescope structure.

The output of the collimator is a $6 \mathrm{~mm}$ diameter beam parallel to the WFS board and a $1300 \times$ compressed image of the pupil reimaged on the surface of a piezo-actuated mirror (2). These mirrors are used to compensate for any residual beam jitter. The integrated tip-tilt command is periodically offloaded from the piezo mirrors to the laser pointing system, to compensate for possible slow drifts of the LGS launch and imaging systems. These piezo mirrors are coupled with flat mirrors in a periscope configuration that allows to reduce the distance between the 3 LGS beams, and therefore the diameter of the on-axis optics in the final part of the WFS, while it allows to keep the output beams parallel to the board plane.

The next group of optics composes the gating unit of the WFS (3). They are an adaptation of the traditional Pockels cell design developed at MPE. In between the 2 linear polarizers there are 2 separate electro-optical crystals and a quartz retarder able to extend the usable field of view up to $\pm 4^{\circ}$ with a clean and ringing free switching behavior with typical time response $<10 \mathrm{~ns}$.

The first lens of the relay optics (4 in figure 2) refocuses the collimated light coming from the Pockels cell in a $F / 33$ beam and it allows to vary both the position of the beam in the pupil and focal planes. Being placed almost in correspondence of the $F / 33$ plane the second lens (5) acts like a field lens allowing to move the pupil position 


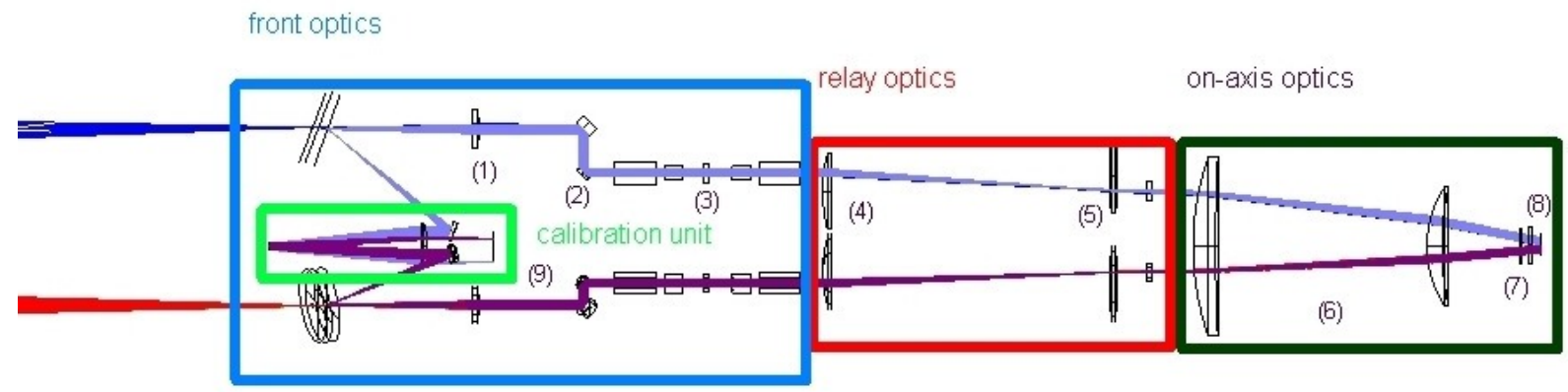

Figure 2. Optical layout of the LGS WFS seen from side, the path of the 2 LGS at the bottom of the triangle are overlapping. In color are shown the main parts that compose the WFS: light blue box highlights the front optics used to independently collimate (1), stabilize (2) and gate (3) the 3 LGS. The red box shows the relay optics that steers the 3 beams toward the optics in common to the whole 3 LGS (black box) on the rear of the WFS. Displacing in the XY plane ( $\mathrm{Z}$ is along the optical axis) the 2 lenses $(4$ and 5$)$ it is possible to position the 3 beams on the LA (7) and CCD plane (8). Two large meniscus lenses (6) collimate the laser beams to feed the $3 \mathrm{SH}$ wavefront sensors. In the middle of the 3 LGS arms on the front part of the WFS it is installed an internal calibration unit (9) to perform the laboratory test and to check for the optics alignment at the telescope. The 3 acquisition cameras are not shown in this picture.

while keeping fixed the spot position on the focal plane. These 2 lenses are held on a support manually adjustable in the XY plane over a $\pm 15 \mathrm{~mm}$ range with respect their nominal position. This large adjustment range is needed to compensate for possible misalignments caused by the birefringence effects in the Pockels cell and to account for the use of a commercial test camera instead of the scientific grade PnCCD during the alignment procedure. In the rear part of the WFS the on-axis optics include a large collimator (6) made of 2 positive menisci lenses with an equivalent focal length of $60 \mathrm{~mm}$ and mounted in a common barrel, a $24 \mathrm{~mm}$ diameter array of square lenslets (7) with $0.384 \mathrm{~mm}$ pitch and $12.3 \mathrm{~mm}$ focal length and the scientific camera (8) holding a pnCCD chip with $248 \times 256 p x$ of $48 \mu m$ size produced by PnSensor Gmbh and developed by MPE. ${ }^{7}$ In this configuration a single WFS subaperture is sensed with $8 \times 8 p x$ equivalent to have a scale of $0.58^{\prime \prime} / p x$ on sky.

An additional element of the LSG-WFS is an internal light source and calibration unit (9) that is described in detail in section 2.1 .

\subsection{WFS internal calibration unit}

The WFS internal calibration unit acts as a telescope simulator during the laboratory test of the WFS, allowing to feed 3 light beams into the WFS and providing a pupil conjugate plane where it can be installed a MEMS-DM. This element will also be used at the telescope, to check the proper alignment of the WFS optics. In this latter case the MEMS-DM will be replaced by a flat mirror.

Figure 3 shows a detail of the optical layout of the WFS internal calibration unit. The light is fed into it by 3 optical fibers (1). Using different fiber core diameters it is possible to simulate for different LGS spot dimensions on-sky. A single double-pass lens (2) first collimates the 3 fiber beams that are reflected backward to it by a mirror (3). A $4.5 \mathrm{~mm}$ diameter pupil stop is overlapped to this mirror, allowing the double-pass lens to return $f_{16}$ beams. A set of fold mirrors adjustable in tip-tilt (4) allow to pick off the 3 beams from the calibration unit and to feed them into the WFS. Figure 3 shows a prototype of calibration unit realized by the Arcetri workshop and installed into the first of LGS-WFS assembled.

\section{WFS DM OPTICAL CALIBRATION}

To perform the closed loop test of the 2 LGS-WFS units a MEMS-DM will be replaced to the flat mirror (3) in figure 3. This device is a Boston Micromachines MULTI-DM having 140 actuators with a $5 \mu m$ stroke and $0.45 \mathrm{~mm}$ inter-actuators pitch. Since this kind of devices lacks of an internal metrology system to measure the actual displacement of the actuators it has been necessary to calibrate and test the accuracy obtainable in the open loop control of this device. ${ }^{9}$ To perform this test the DM was installed on an optical bench and the shape of 


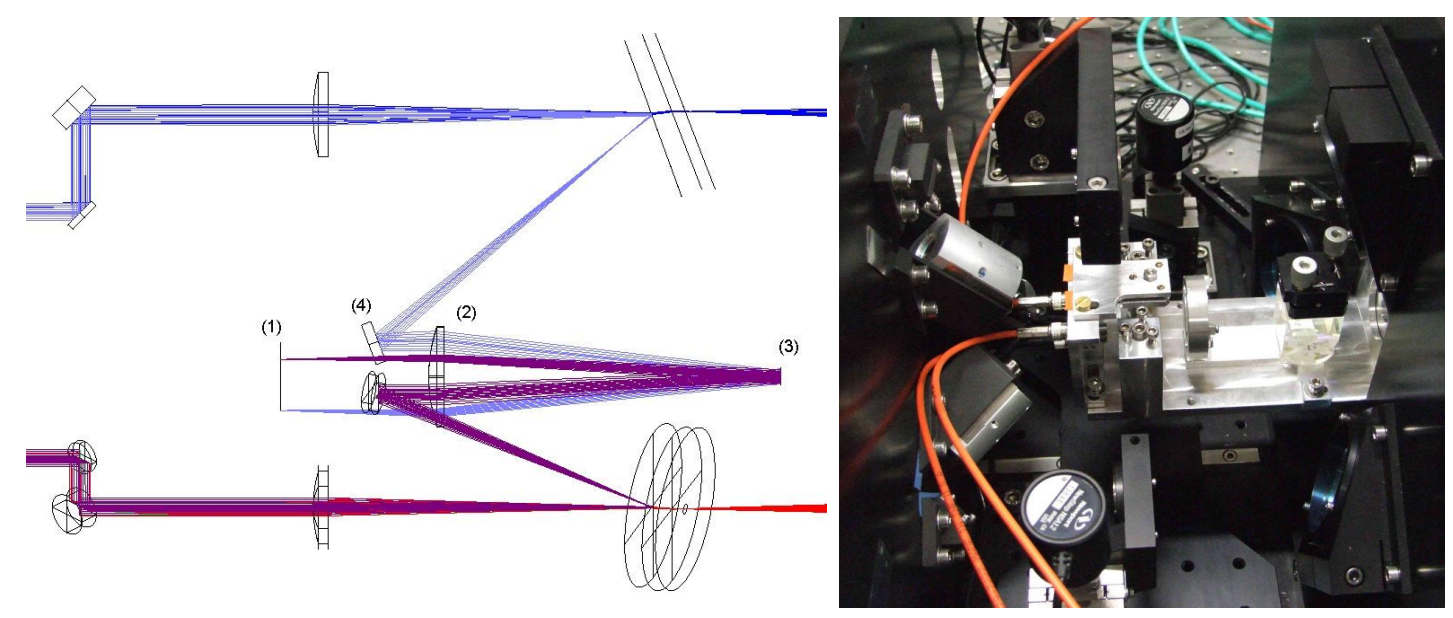

Figure 3. Left: optical layout of the LGS-WFS internal calibration unit seen from side. Light is feeded by 3 optical fibers (1) and it is collimated by a double-pass lens (2). A flat mirror (3), or a MEMS-DM, can be installed on a pupil-conjugate plane on extreme right of the image, back-reflecting light toward the lens that produces $3 f_{16}$ beams. Three adjustable fold mirrors (4) allow to direct the calibration unit light toward the WFS entrance window feeding light into the WFS. Right: prototype of calibration unit realized by the Arcetri workshop and installed on the first unit of WFS assembled in the lab. A cube beamsplitter has been positioned just in front of the DM to direct a collimated laser beam on it having an optical feedback of the DM shape during the WFS closed loop test.

the reflective membrane of the DM was measured using a 4D interferometer (model PhaseCam 4020). The test beam produced by the PhaseCam interferometer has a diameter of $(6.9 \pm 0.1) \mathrm{mm}$, sampled with $990 \times 998 \mathrm{px}$. With this direct imaging setup it has been possible to sample the active area of the DM with a resolution better than $10 \mu \mathrm{m} / p x$.

To perform the WFS test a circular pupil must be overlapped onto the DM square-like reflective area. Since the light beams have an angle of incidence on the DM surface of $\sim 6^{\circ}$ to avoid a mismatching between the actuators pattern seen by the $3 \mathrm{SH}$ sensors the pupil stop must be held at a maximum $0.3 \mathrm{~mm}$ distance from the DM membrane. So a custom made pupil stop has been manufactured and installed on the DM. The measured clear aperture is $(4.50 \pm 0.07) \mathrm{mm}$. This allows to have 10 actuators across the pupil diameter and $\sim 80$ independent actuators within the pupil area while a set of 32 actuators placed along the pupil edge are controlled as slave actuators.

\subsection{Calibration of the actuators response}

The first step has been to measure with the $4 \mathrm{D}$ the displacement of each actuator as a function of voltage commands. From these measures we calibrated the response of the actuators to a given voltage fitting the recorded data with a quadratic law. ${ }^{8}$ The mirror was initially set in the flat state using the reference voltages provided by Boston Micromachines and we swept one actuator at a time on its full range of voltages. The graph in figure 4 shows an example of the measured displacement as a function of a voltage applied in the $0-280 \mathrm{~V}$ range: the $0-200 \mathrm{~V}$ range can be safely used for the mirror operation obtaining an effective stroke of $2 \mu \mathrm{m}$ for a single actuator displacement (this stroke can be increased up to $4 \mu \mathrm{m}$ if a set of many adjacent actuators are moved together). Fitting the measured data with a quadratic law, inverting and solving this function for positive voltage values it is possible to obtain a function that gives the voltage that must be applied to a single actuator to move it in a desired position.

\subsection{Optical flattening and modal basis projection}

Once the volt to displacement relation of each actuator is known we measured the zonal influence functions of the mirror. These are evaluated moving each actuators by a symmetric amount in the positive and negative direction with respect to a default flat position. The measurements correspondent to the positive and negative displacement have been subtracted and divided by the full stroke. 

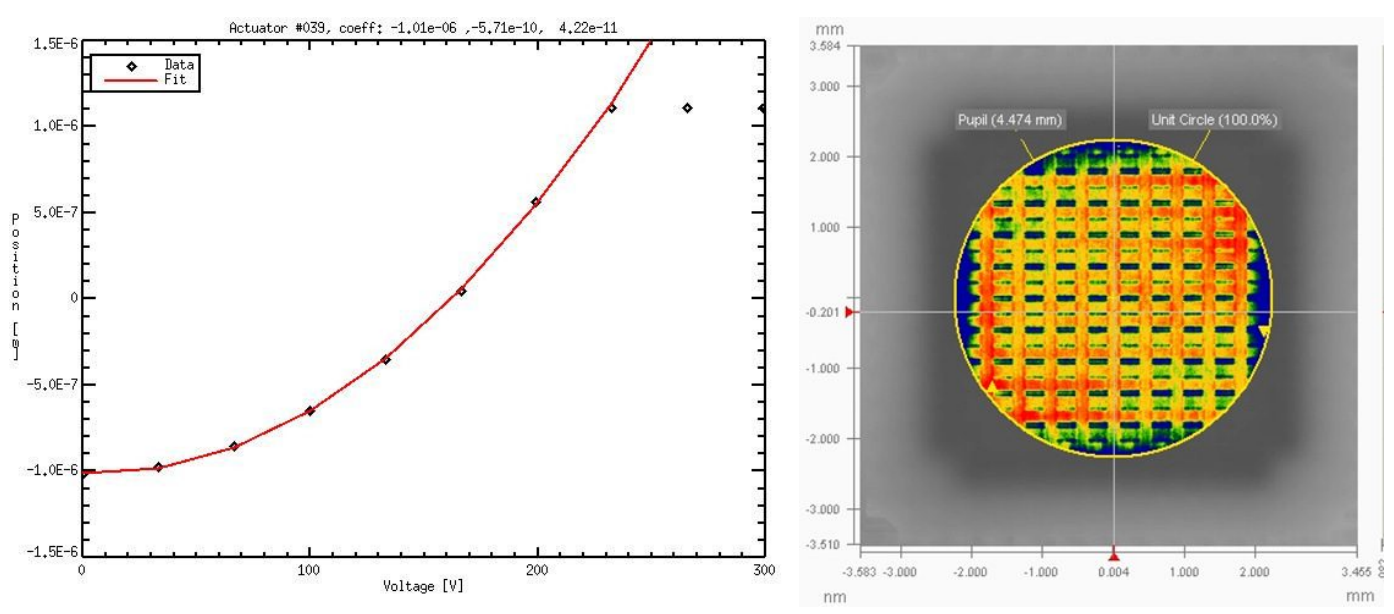

Figure 4. Left: measured displacement of a central actuator to an increasing set of voltages. The quadratic fit performed on the data is shown by the red line. Right: residual surface error of the MEMS-DM after the optical flattening loop performed on the influence functions with the interferometer. In dark gray is visible the DM's square active area while colors highlight the $4.5 \mathrm{~mm}$ clear aperture selected. A residual surface error PtV of $180 \mathrm{~nm}$ and surface rms of $20 \mathrm{~nm}$ (40 $\mathrm{nm}$ wavefront $\mathrm{rms}$ ) has been measured inside the pupil area.

Having the DM influence functions allowed us to operate an optical loop between the interferometer and the $\mathrm{DM}$ to determine the best mirror flat figure. On the $4.5 \mathrm{~mm}$ clear aperture of the DM we measured a residual surface error PtV of $180 \mathrm{~nm}$ corresponding to a surface rms of less than $20 \mathrm{~nm}$, mainly due to the high spatial frequency pattern of the actuators as it can be seen from figure 4 . The voltages applied to set the mirror in this flat position have been recorded and are used as reference position in the closed loop test.

The DM influence functions are required also to project a modal basis on the DM actuator pattern obtaining a modes to volts relation for each actuator. A set of 66 Karhunen-Loève (KL) modes have been considered. We then tested the accuracy in the DM response to a given modal command for varying amplitudes (100, 200, 500 and $1000 \mathrm{~nm} \mathrm{rms}$ ) measuring the fitting error, i.e. the difference between the commanded shape and the one measured with the interferometer. Figure 5 shows the fitting error expressed as WF error rms normalized to the commanded amplitude. Each mode can be reproduced with a $5 \%$ accuracy or better for amplitudes up to $200 \mathrm{~nm} \mathrm{rms}$ (apart from mode 62 as shown in figure 5).

\subsection{Atmospheric turbulence simulation}

To inject a common disturb in the WFS and PSF camera systems we used the DM itself, using a procedure already proved during the First Light AO system acceptance test performed at Arcetri premises in 2010. ${ }^{10}$ Following this procedure, a time evolving turbulence history is generated as a 2D phase screen with a given $r_{0}$ and $L_{0}$ values. Then at each time step, that in this case is fixed to $1 \mathrm{~ms}$, part of the phase screen is extracted accordingly to wind speed, it is projected onto the modal basis and it is applied to the DM.

We tested the amplitude of the atmospheric turbulence that can be injected with the MEMS-DM properly scaling the turbulence history to represent different $r_{0}$ values and measuring with the interferometer the shape effectively set by the DM. We decided to perform all the tests filtering out the tip-tilt component from the atmospheric turbulence disturb, since in the normal LGS-WFS operation the atmospheric tip-tilt will be sensed and corrected independently by a quad-cell Tip-Tilt sensor using a natural guide star.

Figure 6 demonstrate the capability of the MEMS-DM to act as an atmospheric turbulence generator. The curves show the modal wavefront rms amplitude as a function of KL-mode number. We simulated 3 different $r_{0}$ values: 5,10 and $20 \mathrm{~cm}$. The outer scale is $30 \mathrm{~m}$ and the tip-tilt amplitude has been set to zero. For each of the 3 cases 100-400 independent phase screens have been projected on the KL modal basis (black lines) and applied to the MEMS. The resulting figure has been measured with the interferometer and also projected on the KL modal basis (red squares). The blue solid line plotted for reference is the expected spectrum for a Von Karman turbulence with $r_{0}=10 \mathrm{~cm}$ and $L_{0}=30 \mathrm{~m}$. The agreement between commanded mirror shapes and 

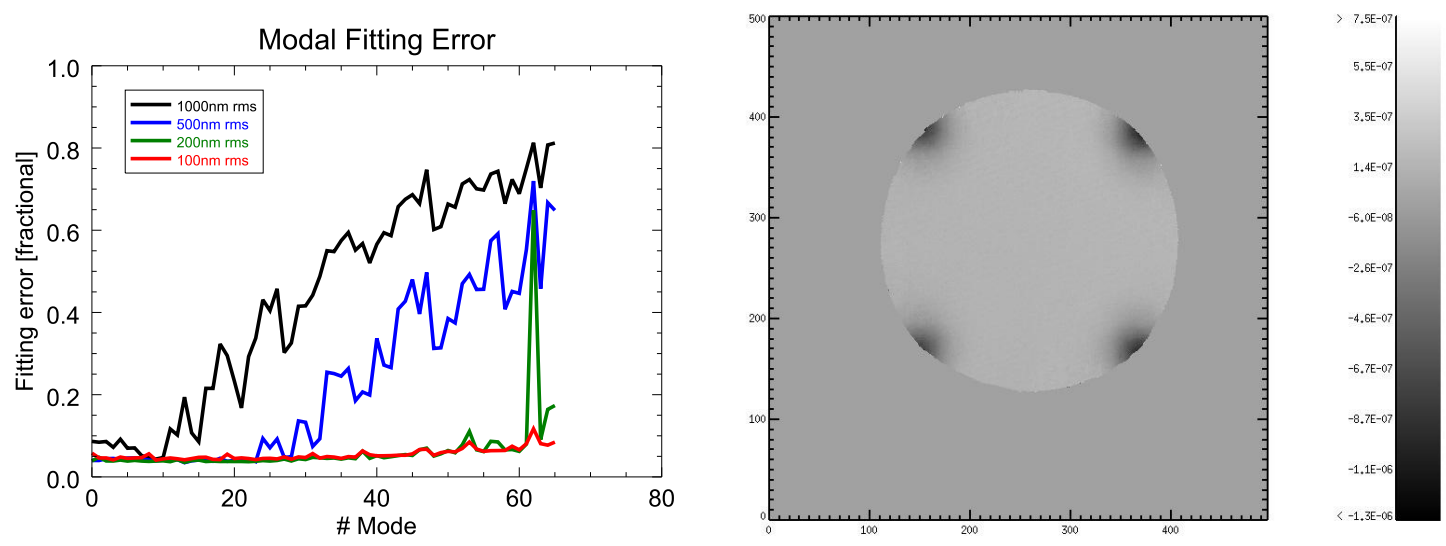

Figure 5. Left: fitting error measured as difference between the WF expected from a modal command applied to the DM and the WF measured by the interferometer. The error values have been normalized to the 4 amplitudes values applied $(100,200,500$ and $1000 \mathrm{~nm} \mathrm{rms})$. The residual rms over the pupil pattern has been plotted for each mode and amplitude. Right: difference between the measured DM shape and the $200 \mathrm{~nm}$ rms command applied to mode 62 , the one corresponding to the peak in the green curve on the left. It is clearly visible that the error is due to the 4 actuators close to the DM corners that are not able to apply the required stroke.
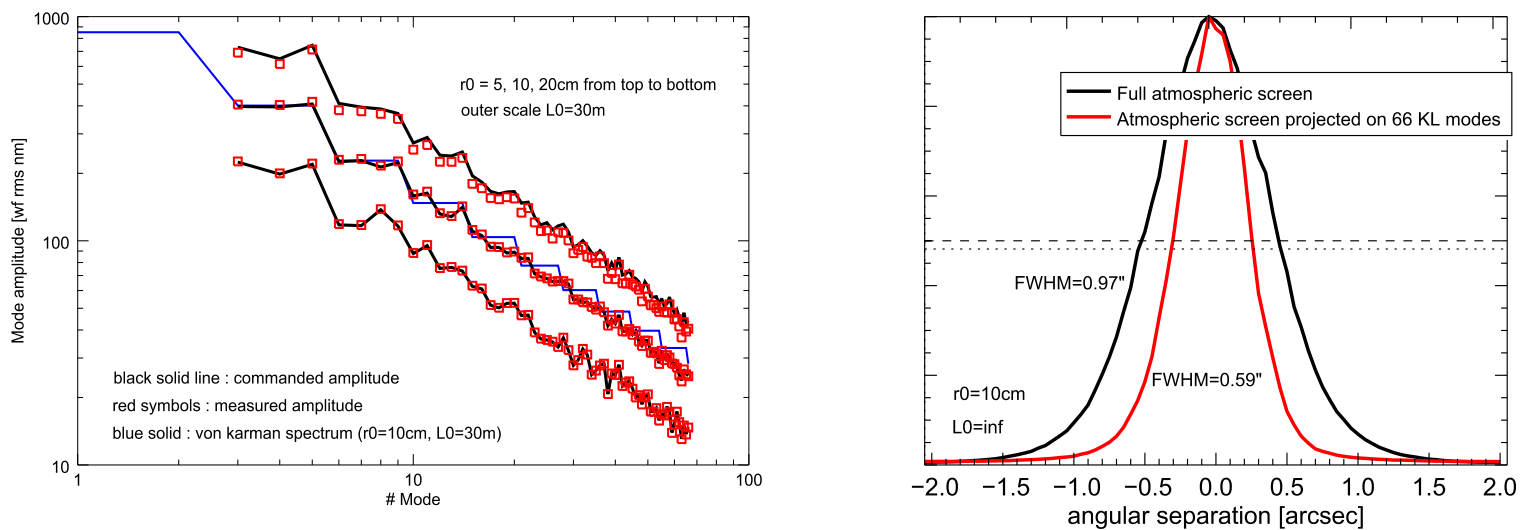

Figure 6. Left: Wavefront rms values of a simulated turbulence history projected onto the 66 KL modal basis and applied to the DM (black lines). The 3 lines refer to different $r_{0}$ values of $0.05,0.1$ and $0.2 m$ respectively. The blue solid line shows for comparison the WF rms value of the Von Karman turbulence spectrum evaluated at $r_{0}=0.1 \mathrm{~m}$ and $L_{0}=30 \mathrm{~m}$. The red squares are the modal WF rms values measured with the interferometer. More details are given in the text. Right: CAOS-simulated seeing-limited PSF created with a $66 \mathrm{KL}$ modes turbulence compared to a full atmospheric screen: the lack of high spatial frequencies results in a narrower PSF. Average of 100 independent phase screens simulated with CAOS with $r_{0}=0.1 m$ and $L_{0}=\infty$

actual interferometer measurements is very good until $r_{0}$ values of $10 \mathrm{~cm}$ : for $r_{0}=5 \mathrm{~cm}$ the effects of the limited stroke of the DM starts to becoming evident.

Since the atmospheric screen is filtered and only the low spatial frequencies corresponding to the 66 KL-modes are transmitted, one may expect a different shape in the seeing limited PSF that can be mimicked in this way. To quantify this effect, we simulated a large set of independent phase screens using CAOS $^{12}$ and we produced a well-averaged long-exposure PSF with or without projection over the MEMS KL basis. Results are shown in Fig. 6 for the case corresponding to $r_{0}=10 \mathrm{~cm}$ and $L_{0}=\infty$ : the PSF simulated with the MEMS 66-modes basis has a FWHM of $0.59^{\prime \prime}$, narrower than the one obtained with the non-filtered screens $\left(0.97^{\prime \prime}\right)$. In the $L_{0}=30 \mathrm{~m}$ case the two values decrease to $0.53^{\prime \prime}$ and $0.83^{\prime \prime}$ respectively. 


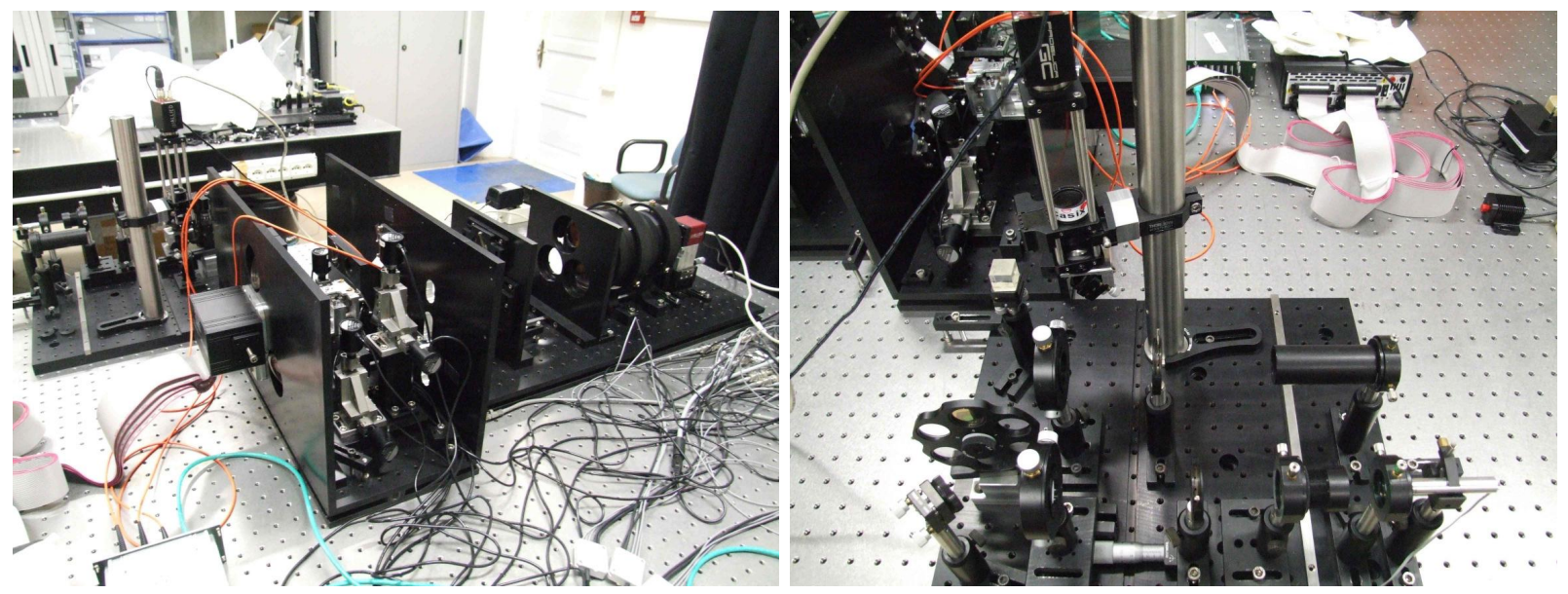

Figure 7. Left: picture of the first unit of LGS-WFS assembled and tested in laboratory. Almost all the final components are present apart the camera and the gating units that have been replaced with a commercial AVT/Prosilica GE2040 camera and by glass rod mimicking the Pockels cells optics. Right: optics used to produce an on-axis diffraction limited PSF at $532 \mathrm{~nm}$ on a dedicated camera (AVT/Prosilica GC1350) to create a PSF during the tests of the WFS.

\section{LABORATORY TEST SETUP AND RESULTS}

The test described in this section have been performed on the first unit of LGS-WFS assembled in laboratory. Figure 7 shows this unit on the optical bench once the internal calibration unit, equipped with the MEMS-DM have been installed. All the key WFS elements described in section 2 are present. The collimating lenses that provide the pupil stabilization on the LA are remotely controllable using Newport NSA12 stepper motors driven by an MPIA MoCon motor controller. The tip-tilt compensation is operated by the piezo-driven mirrors (Physik Instrumente S334.1SL) controlled by the Microgate High Voltage Controller installed in the BCU. At the moment of writing we still miss to integrate in the WFS the final pnCCD camera and the gating units. These 2 elements are replaced temporarily by a test camera (AVT/Prosilica GE2040) and by 3 glass rods mimicking the Pockels cells OPD. The AVT GE2040 detector has $2048 \times 2048$ pixels of $7.4 \mu \mathrm{m}$ with a total size close to the one of the pnCCD, but with a non-integer ratio between the $384 \mu \mathrm{m}$ pitch of the lenslet array and the pixel size.

To evaluate the close-loop performance we installed a beamsplitter in the WFS calibration unit, just in front of the DM as shown in figure 3. In this way it has been possible to direct a collimated laser beam toward the DM and to produce an image on a separate camera. The optics used to produce this on-axis PSF are shown in figure 7. Using a $120 \mathrm{~mm}$ positive lens to produce the image on a AVT/Prosilica GC1350 camera $(4.65 \mu \mathrm{m} / p x)$ and using a $532 \mathrm{~nm}$ beam, we obtained an on-sky pixel scale of $4 \mathrm{mas} / \mathrm{px}$, corresponding to a PSF sampling of $\sim 4 p x$ at the diffraction limit.

Figure 8 shows the arrangement of the $3 \mathrm{SH}$ sensors spots on the test camera (binned by $2,14.8 \mu \mathrm{m} / p x$ ). For the tests described in section 4.2 , we used $40 \mu \mathrm{m}$ core multimode fibers as light sources in the calibration unit. This setup allows to obtain diffraction limited spots on the lenslets image plane corresponding to an equivalent object FWHM of $\sim 100$ mas on sky. At the moment, centroids are computed on $12 \times 12 p x$ areas, corresponding to an on-sky FoV of $2.3^{\prime \prime}$. Figure 8 shows the reference slopes computed when the best flat shape is applied to the DM. The peak-to-valley of the reference slopes amounts to $\sim 0.8^{\prime \prime}$. The main contributor to this large slope $\mathrm{PtV}$ is the astigmatism introduced by the off-axis double pass of light into the calibration unit lens (see figure 3). The defocus term is due to the beamsplitter cube added into the calibration unit and not considered in the optimization of the optical layout. An additional slight defocus is due to the non integer ratio between lenslet size and camera pixel size.

To calibrate the $3 \mathrm{SH}$ sensors, recording their interaction matrices (IM), we used a conventional push-pull technique scaling the modal amplitude of each mode to keep the DM fitting error within the $5 \%$ accuracy level (see figure 5).

Using a pseudo-inversion algorithm and inverting separately the $3 \mathrm{IM}$ recorded it is possible to calibrate each sensor as a SCAO system. In alternative the $3 \mathrm{IM}$ can be concatenated and inverted together to calibrate the 3 

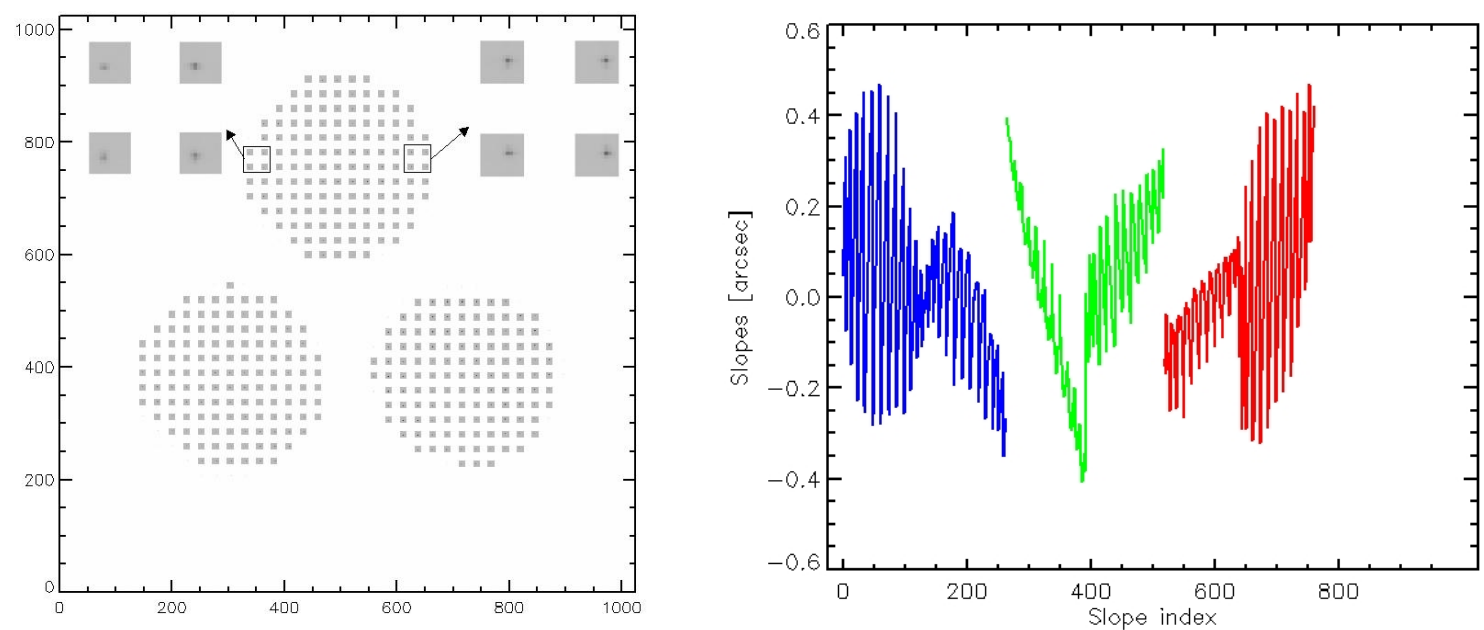

Figure 8. Left: image of the $3 \mathrm{SH}$ spots pattern taken with the alignment camera (binned 2). The subapertures defined via software are highlighted in gray. They have been reduced to a $12 \times 12 p x$ area to reduce the centroiding noise. The two $4 \times 4$ subapertures enlargements on the top right and left show the not perfect centering of the spots over the edge subapertures caused by the not integer ratio between pixel and lenslet size. Right: reference slopes evaluated on the spot pattern shown on left. The slopes $\mathrm{PtV}$ is around $\simeq 0.8^{\prime \prime}$ dominated by an astigmatism term introduced by the off-axis double pass of light into the calibration unit lens.
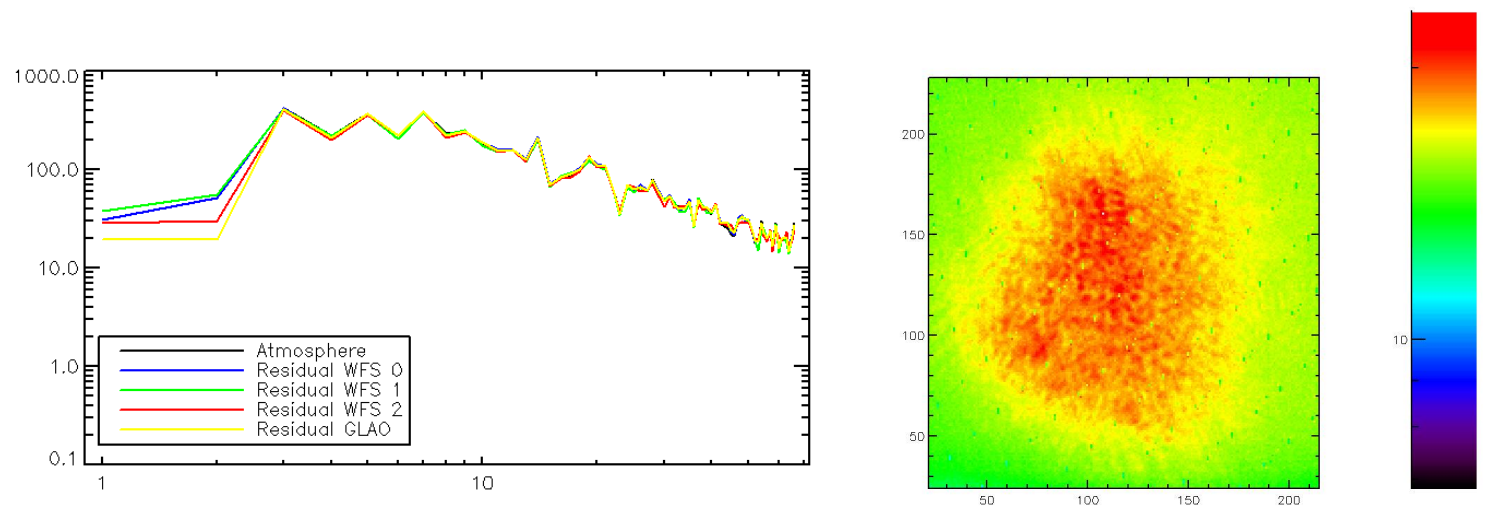

Figure 9. Left: modal rms values of the wavefront disturb injected by the DM and measured by the 3 sensors when calibrated as 3 independent SCAO systems and when calibrated as a GLAO system. Right: long exposure image obtained on the PSF camera averaging a time evolution of $1 \mathrm{~s}$ of the disturb equivalent to a $r_{0}=0.1 \mathrm{~m}$. The measured $\mathrm{FWHM}$ is $0.5^{\prime \prime}$, in good agreement with the CAOS-simulated value of $0.53^{\prime \prime}$.

WFS as a GLAO system.

\subsection{Open loop test}

To check the functionality of the 3 sensors we measured the wavefront disturb injected on the DM independently with the 3 sensors in SCAO mode and together combining the 3 in GLAO mode. Figure 9 shows the modal rms values of the wavefront disturb injected on the DM and measured by the 3 sensors working independently as SCAO systems and working together in GLAO mode. The difference between the injected and measured disturb is below $40 \mathrm{~nm} \mathrm{rms}$ far all cases. On the right of figure 9 it is visible the 1s long-exposure image obtained on the PSF camera. 

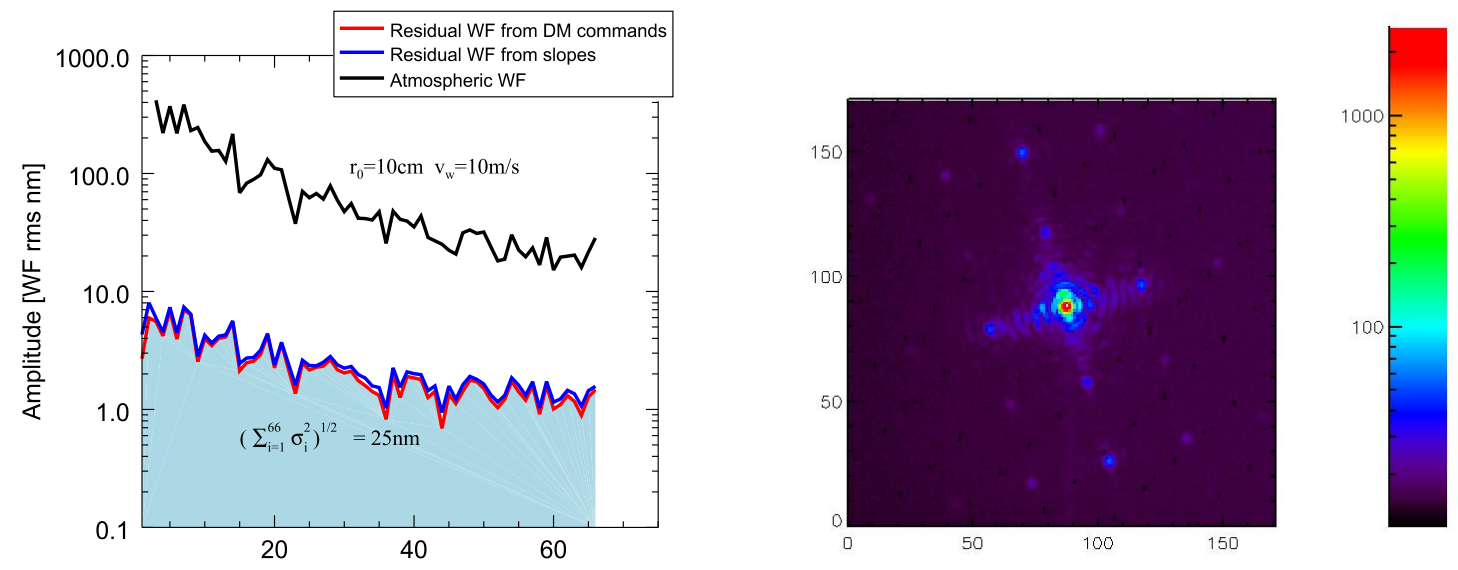

Figure 10. Left: Standard deviation of modal amplitudes for a GLAO close loop in the lab. Atmospheric disturbance is introduced via the DM and contains only the projection on the 66-modes basis that is also used for adaptive correction. Simulated loop frequency $=1 \mathrm{~ms}$, windspeed $=10 \mathrm{~m} / \mathrm{s}$, high-flux regime. Note how the residual wavefront estimated from the DM commands is in very good agreement with the corresponding estimation from WFS slopes. The small discrepancy $(<3 \mathrm{~nm} \mathrm{rms})$ may be due to spurious disturbances (e.g. convection in the lab). Right: long exposure image obtained on the PSF camera $(532 \mathrm{~nm}, 4 \mathrm{mas} / \mathrm{p} x)$ when the GLAO loop is closed for a time evolution equivalent to $1 \mathrm{~s}$. The square pattern is due to the geometry of the DM. The Strehl ratio is $71 \%$.

\subsection{Closed loop test}

Since the turbulence disturbance is applied only on the pupil conjugate mirror without mimicking any high altitude layer, the GLAO system in these tests should be able to measure and correct all the injected turbulence, allowing to obtain a diffraction limited image on the PSF camera even with only 66 corrected modes. Note also that in this case the control loop commands are opposite to the turbulence commands, resulting in a global DM figure close to the flat shape: as a consequence of that, the DM command history in close loop can be used to estimate the residual WF error.

Figure 10 resumes the results of the close loop tests: the black line shows the WF modal rms values for the artificial atmospheric perturbation (the one plotted also in figure 9). The color lines show the residuals obtained in GLAO mode evaluated in two independent ways: from WFS slopes (blue line) and from DM total commands, i.e. the difference between artificial disturbance and control loop command (red line). The WF residual integrated on the 66 controlled modes amounts to $25 \mathrm{~nm} \mathrm{rms}$. The small discrepancy between blue and red line $(<3 \mathrm{~nm}$ rms) may be due to spurious disturbances (e.g. convection in the lab) that are not taken into account in the red line case, where the artificial turbulence is considered as the only input to the loop.

The Strehl ratio (SR) for the PSF has been evaluated using the same routines used during the commissioning of the FLAO system. We first set the MEMS DM in the shape that corresponds to the minimized static aberration in the PSF optical path, using an iterative algorithm to determine the best value of the first 15 modes. The SR estimated on a PSF obtained in such a way is $80 \%$ at $532 \mathrm{~nm}$ corresponding to a WF aberration of $40 \mathrm{~nm}$ in Marechal's approximation. This is in perfect agreement with the interferometric measurement of the best wavefront described in section 3.2 and demonstrate that non-common path aberration have been corrected.

When the adaptive loop is closed, the SR decreases to $71 \%$ (see figure 10), corresponding to a residual wavefront error of $49 \mathrm{~nm}$. This is also in very good agreement with a budget error made up of the two main contribution discussed so far: $25 \mathrm{~nm}$ due to control loop errors, $40 \mathrm{~nm}$ due to high-spatial frequencies aberrations in the "best flat" shape that are outside of the corrected modal basis. A second test that has been done is the comparison of the performance of the WFS when used as GLAO sensor and when each of the 3 arms is used as SCAO sensors. Since the only disturbance is applied in the pupil plane we can expect similar performances in the 4 cases. Figure 11 shows a comparison of the modal residuals: the wavefront residual integrated over the 66 controlled modes amount to 30,36 and $27 \mathrm{~nm}$ rms for the 3 independent SCAO systems, slightly higher than the $25 \mathrm{~nm}$ of the GLAO case. 


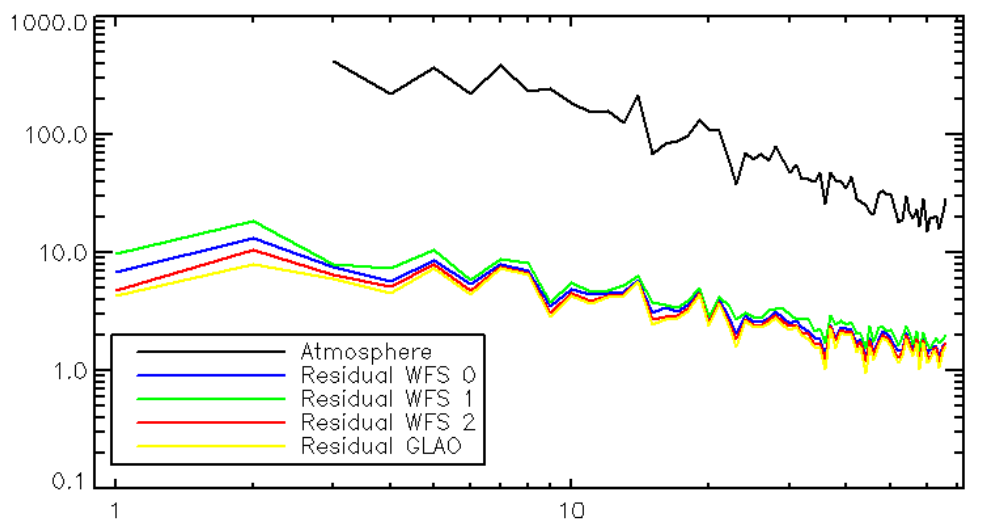

Figure 11. Left: modal rms values of the wavefront disturb injected on the DM and of the AO correction residuals. The different lines refer to the loop closed on the 3 sensors when calibrated independently as SCAO systems and when calibrated as a single GLAO system

\section{CONCLUSIONS}

We have presented some preliminary results of the ARGOS LGS-WFS performances, obtained during the integration phase in Arcetri's laboratories.

Closed loop test are performed using the internal calibration unit of the LGS-WFS with a ground-conjugated MEMS-DM acting both as corrector and as turbulence generator. A PSF camera producing an image of the corrected on-axis beam allows to evaluate AO loop performances. We tested with this setup the 3 sensors working independently as 3 separate SCAO systems and together in GLAO mode. From the residual slopes, we evaluated the corrected WFE integrated over the 66-modes modal basis to be 30,36 and $27 \mathrm{~nm}$ rms for the 3 SCAO WFS in high-flux regime and mimicking a $1 \mathrm{kHz}$ loop rate and $r_{0}=0.1 \mathrm{~m}$. The same test performed using the 3 WFS together in a GLAO system gave a residual WFE of $25 \mathrm{~nm} \mathrm{rms}$. These values are backed up by the SR evaluated on the GLAO PSF image.

\section{REFERENCES}

1. S. Rabien, N. Ageorges, L. Barl, et al., ARGOS: the laser guide star system for the LBT, Proc. SPIE 7736, $77360 \mathrm{E}(2010)$.

2. Walter Seifert et. al., LUCIFER: a Multi-Mode NIR Instrument for the LBT, Proc. SPIE 4841, 962-973 (2003).

3. Lorenzo Busoni, Marco Bonaglia, Simone Esposito, Luca Carbonaro and Sebastian Rabien, Final design of the wavefront sensor unit for ARGOS, the LBT's LGS facility, Proc. SPIE 7736, 77365K (2010).

4. C. Loose et. al.; Testing and integrating the laser system of ARGOS, the Ground Layer Adaptive Optics for $L B T$, Proc. SPIE this proceeding.

5. Roberto Biasi, Mario Andrighettoni, Armando Riccardi, Valdemaro Biliotti, Luca Fini, Armando Riccardi, Paolo Mantegazza, Daniele Gallieni, Dedicated flexible electronics for adaptive secondary control, Proc. SPIE 5490, 1502 (2004).

6. Armando Riccardi, Marco Xompero, Runa Briguglio, Fernando Quiros-Pacheco, Lorenzo Busoni, Luca Fini, Alfio Puglisi, Simone Esposito, Carmelo Arcidiacono, Enrico Pinna, Piero Ranfagni, Piero Salinari, Guido Brusa, Richard Demers, Roberto Biasi, and Daniele Gallieni, The adaptive secondary mirror for the Large Binocular Telescope: optical acceptance test and preliminary on-sky commissioning results, Proc. SPIE 7736, $77362 \mathrm{C}(2010)$.

7. Gilles Orban de Xivry, Sebastian Rabien, Lothar Barl, Simone Esposito, Wolfgang Gaessler, Michael Hart, Matthias Deysenroth, Hans Gemperlein, Lothar Struder, and Julian Ziegleder, Wide-field AO correction: the large wavefront sensor detector of ARGOS, Proc. SPIE 7736, 77365C (2010). 
8. Mark N. Horenstein, Thomas G. Bifano, Raji Krishnamoorthy Mali and Nelsimar Vandelli, Electrostatic Effects in Micromachined Actuators for Adaptive Optics, Journal of Electrostatics 42 69-81 (1997).

9. Thomas G. Bifano, Julie A. Perreault and Paul A. Bierden, Micromachined deformable mirror for optical wavefront compensation, Proc. SPIE 4124, 7 (2000).

10. Simone Esposito, Armando Riccardi, Luca Fini, Alfio T. Puglisi, Enrico Pinna, Marco Xompero, Runa Briguglio, Fernando Quiros-Pacheco, Paolo Stefanini, Juan C. Guerra, Lorenzo Busoni, Andrea Tozzi, Francesca Pieralli, Guido Agapito, Guido Brusa-Zappellini, Richard Demers, Joar Brynnel, Carmelo Arcidiacono, and Piero Salinari, First light AO (FLAO) system for LBT: final integration, acceptance test in Europe, and preliminary on-sky commissioning results, Proc. SPIE 7736, 773609 (2010).

11. Esposito, S., Riccardi, A., Pinna, E., et al., Large Binocular Telescope Adaptive Optics System: new achievements and perspectives in adaptive optics, Proc. SPIE 8149, 814902-814902-10 (2011).

12. M. Carbillet, G. Desidera, E. Augier, A. La Camera, A. Riccardi, A. Boccaletti, L. Jolissaint, D. Al Kabir, The CAOS problem-solving environment: recent developments, Proc. SPIE 7736, 773644 (2010). 